\title{
Seismic Wavelet Estimation: A Frequency Domain Solution to a Geophysical Noisy Input-Output Problem
}

\author{
Andrew T. Walden, Associate Member, IEEE, and Roy E. White
}

\begin{abstract}
In seismic reflection prospecting for oil and gas a key step is the ability to estimate the seismic wavelet (impulse response) traveling through the earth. Such estimation enables filters to be designed to deblur the recorded seismic time series and allows the integration of "downhole" and surface seismic data for seismic interpretation purposes. An appropriate model for the seismic time series is a noisy-input/noisy-output linear model. We tackle the estimation of the impulse response in the frequency domain by estimating its frequency response function. We use a novel approach where multiple coherence analysis is applied to the replicated observed output series to estimate the output signal-to-noise ratio (SNR) at each frequency. This, combined with an estimate of the ordinary coherence between observed input and observed output, and with the spectrum of the observed input and cross-spectrum of the observed input and output, enables estimation of the frequency response function. The methodology is seen to work well on real and synthetic data.
\end{abstract}

Index Terms-Frequency domain analysis, geophysics, identification, signal processing.

\section{INTRODUCTION}

$\mathbf{I}$ $\mathrm{N}$ TIME series analysis of stationary processes, the situation often arises where the input to a linear system is imperfectly observed, as well as the output. Such a situation is shown in Fig. 1 and is often known as the noisy input-output problem [20], [22] or the errors in variables (EIV) problem [11], [15]. $\left\{O_{t}\right\}$ is the observed output series, consisting of the true output series $\left\{y_{t}\right\}$ plus the output noise series $\left\{\eta_{t}\right\} \cdot\left\{D_{t}\right\}$ is the observed input series, consisting of the true input series $\left\{r_{t}\right\}$ plus the input noise series $\left\{\varepsilon_{t}\right\}$. The model is partly specified by

$$
\begin{aligned}
y_{t} & =r * h_{t}=\Delta t \sum_{k=-\infty}^{\infty} h_{k} r_{t-k} \\
O_{t} & =y_{t}+\eta_{t} \\
D_{t} & =r_{t}+\varepsilon_{t}
\end{aligned}
$$

Manuscript received March 1, 1996; revised February 26, 1997.

A. T. Walden is with the Department of Mathematics, Imperial College of Science, Technology, and Medicine, London SW7 2BZ U.K. (e-mail: a.walden@ic.ac.uk).

R. E. White is with the Department of Geology, Birkbeck College, University of London, London WC1E 7HX U.K.

Publisher Item Identifier S 0196-2892(98)00156-9.
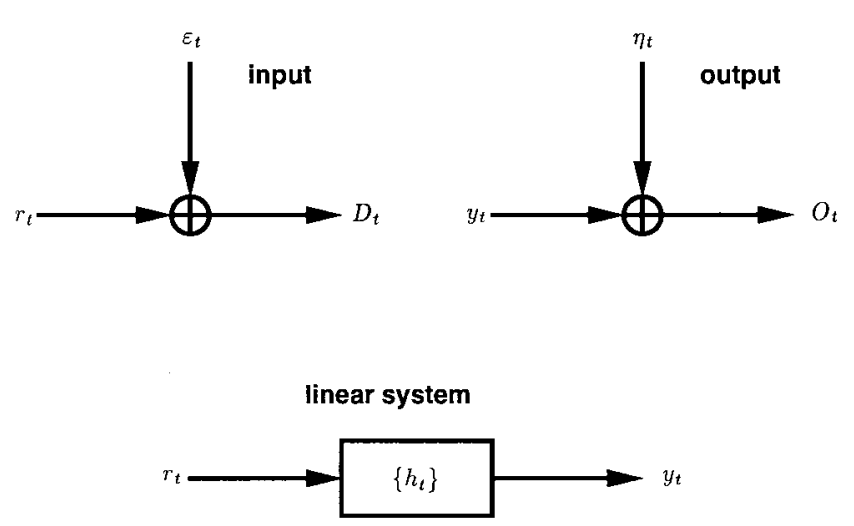

Fig. 1. The errors in variables linear system.

where '*' denotes convolution and $\left\{h_{t}\right\}$ is the impulse response of the filter defining the linear system. The sample interval is denoted by $\Delta t$.

To complete the specification, we must consider the structures of the processes involved. We shall be considering the analysis of reflection seismology data, arising in oil and gas exploration, as discussed in, e.g., [1] and [2]. In oil and gas exploration, the model of Fig. 1 arises as follows. Consider the highly simplified diagram of Fig. 2(a). This represents a marine seismic survey. A ship moves in a straight line on the sea surface and regularly fires off an explosion from an array of airguns. The source signature from each explosion is converted by transmission through the sea, seabed sediments, and upper layers of the overburden into a seismic wavelet which is reflected back from each boundary between the layers of consolidated rock at depth. Associated with each boundary between two layers of rock is a reflection coefficient. The sequence of boundaries going into the earth from a particular surface location are represented by a finely sampled series (equispaced at intervals $\Delta t$ ) of reflection coefficients $\left\{r_{t}\right\}$ for digital computation purposes.

We obviously do not know this sequence at every surface location (it varies slowly with lateral position), but usually in oil and gas exploration a bore-hole will be drilled at a location, $P$ say, of particular interest in a survey; see Fig. 2(b). In this case logging sondes will be placed in the bore-hole and pulled upwards, measuring rock velocity and density of the layers of rock. Since a reflection coefficient is the difference in acoustic impedance of two layers, over their sum, and acoustic 


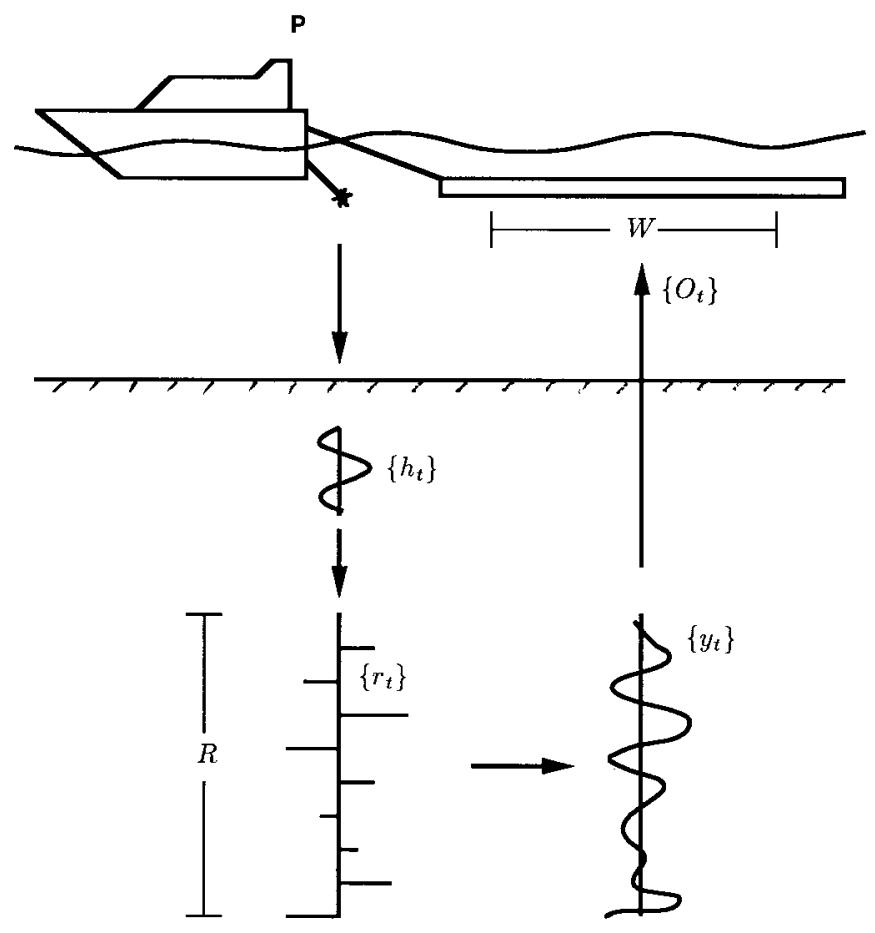

(a)

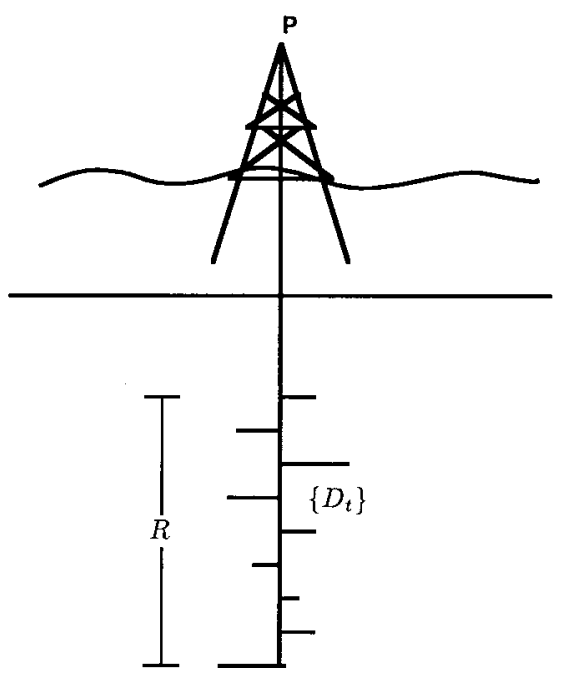

(b)

Fig. 2. (a) Schematic of the seismic acquisition method at a location $P$. After transmission through the shallow sediments beneath the seabed, the pulse generated by the airguns towed by the ship is converted into a seismic wavelet $\left\{h_{t}\right\}$. The observed seismic time series $\left\{O_{t}\right\}$ recorded by a towed hydrophone array over a time window $W$ is the convolution of $\left\{h_{t}\right\}$ with the reflectivity sequence $\left\{r_{t}\right\}$ in some depth range $R$. (b) Measurement of $\left\{D_{t}\right\}$, an approximation to the true reflection sequence $\left\{r_{t}\right\}$, using a well drilled at location $P$.

impedance is given by velocity times density, it is thus possible to construct (observe) a series $\left\{D_{t}\right\}$ which is close to the true reflection sequence $\left\{r_{t}\right\}$, i.e., $D_{t}=r_{t}+\varepsilon_{t}$. The input noise $\left\{\varepsilon_{t}\right\}$ arises due to errors in the physical experiment, due to causes such as extreme conditions in the bore-hole and instrument calibration inaccuracies. The construction of $\left\{D_{t}\right\}$, including the block-averaging for the production of equispaced samples, is discussed, for example, in [3].
Now consider the returning reflections recorded at intervals $\Delta t$ in a cable, full of hydrophones, towed behind the ship, as in Fig. 2(a). Let us denote the recorded ("observed") time series at the location $P$ of particular interest by $\left\{O_{t}\right\}$. Over a specified time window $W$, chosen so that the stationarity assumption is not obviously violated, the recorded series consists of the convolution of the seismic wavelet $\left\{h_{t}\right\}$ active at the corresponding depth range $R$, with the sequence of reflection coefficients in that same depth range $\left\{r_{t}\right\}$ plus noise $\left\{\eta_{t}\right\}$ due to the sea state, recording equipment etc. Hence, we get $O_{t}=y_{t}+\eta_{t}$, where $y_{t}=\Delta t \sum_{k=-\infty}^{\infty} h_{k} r_{t-k}$.

From consideration of the above physical situation, the following assumptions are appropriate.

1) Output noise series $\left\{\eta_{t}\right\}$ is independent of the input series $\left\{r_{t}\right\}$ to the filter and also of the input noise series $\left\{\varepsilon_{t}\right\}$ and independent of the output $\left\{y_{t}\right\}$ of the filter.

2) Input noise series $\left\{\varepsilon_{t}\right\}$ is likewise independent of $\left\{r_{t}\right\},\left\{y_{t}\right\}$ and $\left\{\eta_{t}\right\}$.

3) Series may be analyzed as (second-order) stationary and furthermore, from detailed investigations in [4] and [5], may be taken to have a mean of zero and to have a square summable autocovariance sequence so that their spectra exist in the usual mean square sense.

Estimation of the seismic wavelet $\left\{h_{t}\right\}$, or of its corresponding frequency response

$$
H(f)=\Delta t \sum_{k=-\infty}^{\infty} h_{k} \exp (-i 2 \pi f k \Delta t)
$$

would enable the design of an inverse filter to deblur the recorded traces. Such deconvolution (deblurring) is a vital and ubiquitously used statistical technique in geophysics (e.g., [6], [7]). In this paper we are thus concerned with how we can accurately estimate $\left\{h_{t}\right\}$ or $H(f)$. In Section II, we consider the form of the frequency response function and, in Section III, rewrite this in terms of the ordinary coherence between observed input and output and the output signalto-noise ratio (SNR). This formula forms the basis of our estimation scheme. In Section IV, we contrast our method with other approaches, including classical noisy input-output (EIV) solutions. The novel contribution of our approach lies in taking advantage of the multiple outputs available in the geophysical exploration setting, enabling estimation of the output SNR via multiple coherence of multiple output series. Hence we work just with second-order statistics, and unlike with modern single-input/single-output approaches (e.g., [18], [22]), higher order statistics are not required. Section V gives the equation linking the multiple coherences to the SNR's of the replicated output series; this enables computation of the output SNR at the well location. The estimation at the well location of the output SNR, and of the ordinary coherence between the noisy input and output, leading to estimation of the frequency response function, is discussed in Section VI. Section VII illustrates applications of the method. First, real data is used (an uncontrolled case study) by using a well-log synthetic seismogram $\left\{D_{t}\right\}$ and seismic data $\left\{O_{t, 1}\right\},\left\{O_{t, 2}\right\}$, and $\left\{O_{t, 3}\right\}$ from the southern North Sea. Second, a controlled 
experiment is carried out using simulated data and various SNR's on input and output. The method is seen to work very satisfactorily in practice in both cases.

\section{FREQUENCY RESPONSE ESTIMATION}

If we multiply through $\left\{y_{t+\tau}\right\}$ defined in (1) by $r_{t}$ and take expected values we obtain

$$
s_{\tau, r y}=\Delta t \sum_{k=-\infty}^{\infty} h_{k} s_{\tau-k, r}
$$

where $s_{\tau, r y}=\operatorname{cov}\left\{r_{t}, y_{t+\tau}\right\}=E\left\{r_{t} r_{t+\tau}\right\}$ is the crosscovariance sequence between $\left\{r_{t}\right\}$ and $\left\{y_{t}\right\}$ and $s_{\tau, r}=$ $\operatorname{cov}\left\{r_{t}, r_{t+\tau}\right\}=E\left\{r_{t} r_{t+\tau}\right\}$ is the autocovariance sequence for $\left\{r_{t}\right\}$. Fourier transforming gives the following relationship between the cross-spectrum of $\left\{r_{t}\right\}$ and $\left\{y_{t}\right\}$, denoted by $S_{r y}(f)$, and the spectrum of $\left\{r_{t}\right\}$, denoted by $S_{r}(f)$ :

$$
S_{r y}(f)=H(f) S_{r}(f)
$$

so that the true frequency response function is

$$
H(f)=\frac{S_{r y}(f)}{S_{r}(f)}
$$

Here

$$
S_{r}(f)=\Delta t \sum_{\tau=-\infty}^{\infty} s_{\tau, r} e^{-i 2 \pi f \tau \Delta t} \quad \text { for }|f| \leq \frac{1}{2 \Delta t}=f_{(N)}
$$

is the spectrum for the process $\left\{r_{t}\right\}$, and $f_{(N)}=1 /(2 \Delta t)$ is the Nyquist frequency. Also

$$
S_{r y}(f)=\Delta t \sum_{\tau=-\infty}^{\infty} s_{\tau, r y} e^{-i 2 \pi f \tau \Delta t}, \quad|f| \leq f_{(N)}
$$

is the cross-spectrum of $\left\{r_{t}\right\}$ and $\left\{y_{t}\right\}$. Hence, if $\left\{r_{t}\right\}$ and $\left\{y_{t}\right\}$ were observed without error, the transfer function could be estimated simply using (4). However, we are able to observe only $\left\{D_{t}\right\}$ and $\left\{O_{t}\right\}$ given by (2) and (3). From (3), and the uncorrelatedness of $\left\{r_{t}\right\}$ and $\left\{\varepsilon_{t}\right\}$ and zero mean assumptions, the autocovariance sequence for $\left\{D_{t}\right\}$ is given by $s_{\tau, D}=\operatorname{cov}\left\{D_{t}, D_{t+\tau}\right\}=E\left\{D_{t} D_{t+\tau}\right\}=s_{\tau, r}+s_{\tau, \varepsilon}$, and similarly $s_{\tau, O}=s_{\tau, y}+s_{\tau, \eta}$. The cross-covariance sequence between $\left\{D_{t}\right\}$ and $\left\{O_{t}\right\}$, is $s_{\tau, D O}=\operatorname{cov}\left\{D_{t}, O_{t+\tau}\right\}=s_{\tau, r y}$, where $s_{\tau, r y}$ is the cross-covariance sequence between $\left\{r_{t}\right\}$ and $\left\{y_{t}\right\}$. Fourier transforming these auto and cross-covariance relationships gives the following spectrum relationships

$$
\begin{aligned}
S_{D}(f) & =S_{r}(f)+S_{\varepsilon}(f) \\
S_{O}(f) & =S_{y}(f)+S_{\eta}(f) \\
S_{D O}(f) & =S_{r y}(f) .
\end{aligned}
$$

Clearly, consistent estimators of $S_{D}(f)$ and $S_{O}(f)$ will be inconsistent estimators of $S_{r}(f)$ and $S_{y}(f)$. We can define the filter that predicts $\left\{O_{t}\right\}$ from $\left\{D_{t}\right\}$ in the least squares sense as $H_{O \mid D}(f) \equiv S_{D O}(f) / S_{D}(f)$. This corresponds to the standard Wiener filter approach: divide the cross-spectrum of observed input and output by the spectrum of the observed input. Now

$$
H_{O \mid D}(f) \equiv \frac{S_{D O}(f)}{S_{D}(f)}=\frac{S_{r y}(f)}{S_{D}(f)}=\frac{H(f) S_{r}(f)}{S_{r}(f)+S_{\varepsilon}(f)} .
$$

Hence, if $S_{\varepsilon}(f) \equiv 0$, then $H_{O \mid D}(f) \equiv H(f)$; estimation and accuracy considerations in this case are discussed in detail in [8]-[10]. It is more physically realistic to assume that there is some input noise at every frequency, or $S_{\varepsilon}(f) \neq 0$, so that

$$
\begin{aligned}
H(f) & =\left[1+\frac{S_{\varepsilon}(f)}{S_{r}(f)}\right] \frac{S_{D O}(f)}{S_{D}(f)} \\
& =\left[\frac{1+\rho_{\text {in }}(f)}{\rho_{\text {in }}(f)}\right] \frac{S_{D O}(f)}{S_{D}(f)} \\
& =\left[\frac{1+\rho_{\mathbf{i n}}(f)}{\rho_{\mathbf{i n}}(f)}\right] H_{O \mid D}(f)
\end{aligned}
$$

where $\rho_{\text {in }}(f)=S_{r}(f) / S_{\varepsilon}(f)$ is the input SNR. Hence, in order to estimate $H(f)$ from the observed series $\left\{D_{t}\right\}$ and $\left\{O_{t}\right\}$ we must in general allow for the effect of the input SNR term in the square bracket. Put another way, at every frequency we should modify the Wiener filter corresponding to the observed input and output by multiplying by the unbiasing term $\left[1+\rho_{\mathbf{i n}}(f)\right] / \rho_{\mathbf{i n}}(f)$. We emphasize again that this term is frequency dependent. This is the frequency domain analog to the result that ordinary least squares estimates are biased in the EIV setting (e.g., [11]). The estimation of the impulse response or frequency response function in this situation has been tackled in [12] and [13] where it is assumed that the impulse response is minimum-delay (the frequency response is minimum-phase), often called "invertible" in time series analysis. Due to the many steps in seismic data processing this assumption is usually unrealistic (e.g., [14]), and in this paper we do not make the assumption. Instead, we make use of the replicated nature of seismic time series.

\section{ORDINARY COHERENCE AND SIGNAL-TO-NOISE RATIOS}

The ordinary coherence $\gamma_{D O}^{2}(f)$ or magnitude-squared coherence, which measures the linear correlation between the components of $\left\{O_{t}\right\}$ and $\left\{D_{t}\right\}$ at frequency $f$ is defined as

$$
\gamma_{D O}^{2}(f)=\frac{\left|S_{D O}(f)\right|^{2}}{S_{D}(f) S_{O}(f)}
$$

Using (5)-(7), this can be written

$$
\gamma_{D O}^{2}(f)=\frac{\left|S_{r y}(f)\right|^{2}}{\left[S_{r}(f)+S_{\varepsilon}(f)\right]\left[S_{y}(f)+S_{\eta}(f)\right]} .
$$

Now, since $\left\{y_{t}\right\}$ is the series $\left\{r_{t}\right\}$ convolved with the impulse response $\left\{h_{t}\right\}$, the spectra are related by

$$
S_{y}(f)=|H(f)|^{2} S_{r}(f) .
$$

Using (4) and (10), we get $\left|S_{r y}(f)\right|^{2}=S_{r}(f) S_{y}(f)$, so that (9) can be written

$$
\begin{aligned}
\gamma_{D O}^{2}(f) & =\frac{S_{r}(f) S_{y}(f)}{\left[S_{r}(f)+S_{\varepsilon}(f)\right]\left[S_{y}(f)+S_{\eta}(f)\right]} \\
& =\left[\frac{\rho_{\text {in }}(f)}{1+\rho_{\text {in }}(f)}\right]\left[\frac{\rho_{\text {out }}(f)}{1+\rho_{\text {out }}(f)}\right]
\end{aligned}
$$


where $\rho_{\text {out }}(f)=S_{y}(f) / S_{\eta}(f)$ is the output SNR (again assuming physical reality, i.e., there is some noise at every frequency or $S_{\eta}(f) \neq 0$ ). Note that if $\rho_{\text {out }}(f)=0$, but $\rho_{\text {in }}(f) \neq 0$ at some frequency, then $\gamma_{D O}^{2}(f)=0$ and from (9), $\left|S_{D O}(f)\right|=0$, so that from (8), $|H(f)|=0$, i.e., the frequency response, "by definition," has no amplitude at that frequency. Finally, from (8),

$$
H(f)= \begin{cases}\frac{1}{\gamma_{D O}^{2}(f)}\left[\frac{\rho_{\text {out }}(f)}{1+\rho_{\text {out }}(f)}\right] \frac{S_{D O}(f)}{S_{D}(f)}, & \text { if } \rho_{\text {out }}(f)>0 \\ 0, & \text { otherwise }\end{cases}
$$

which will form the basis of our estimation scheme.

\section{OTHER POSSIBLE APPROACHES}

It is shown in Appendix A that the nonnull case in (12) can be written as

$$
H(f)=\frac{\left[\lambda(f) S_{O}(f)-S_{D}(f)\right]+U(f)}{2 \lambda(f) S_{D O}^{*}(f)}
$$

where

$$
U(f)=\sqrt{\left[\lambda(f) S_{O}(f)-S_{D}(f)\right]^{2}+4 \lambda(f)\left|S_{D O}(f)\right|^{2}}
$$

and $\lambda(f)$ denotes the ratio of input noise spectral density function to output noise spectral density function at frequency $f$, i.e., $\lambda(f)=S_{\varepsilon}(f) / S_{\eta}(f)$, and $\sqrt[+]{ } \cdot$ denotes positive square root. In this paper we will estimate $H(f)$ using (12) rather than (13). Hence, we avoid the variance ratio $\lambda(f)$ and instead estimate the SNR $\rho_{\text {out }}(f)$ and the ordinary coherence $\gamma_{D O}^{2}(f)$. With replicated output series $\rho_{\text {out }}(f)$ can be estimated using multiple coherence analysis, while $\gamma_{D O}^{2}(f)$ can be estimated using ordinary coherence analysis. In estimating $\rho_{\text {out }}(f)$ and $\gamma_{D O}^{2}(f)$ we use smoothed spectral estimates with smoothing window bandwidth $B_{W}$. (Note that use of raw, unsmoothed, periodogram ordinates makes the coherence exactly unity.) Hence, with respect to estimation at the Fourier frequencies $f_{k}=k /(N \Delta t)$ for integers $k$ such that $|k| \leq\lfloor N / 2\rfloor$, the relation $Y(f)=H\left(f_{k}\right) X(f)$ is implicitly assumed to apply for frequencies $\left\{f: f_{k}-\left(B_{W} / 2\right)<f<f_{k}+\left(B_{W} / 2\right)\right\}$, which is a smoothness assumption. In other words, the estimate gets its degrees of freedom from contiguous spectral ordinates within the smoothing window bandwidth.

An alternative approach is to use classical EIV solutions. If we apply the discrete Fourier transform to (1)-(3) for a segment $t=0, \cdots, N-1$, we obtain, at the Fourier frequencies

$$
\begin{aligned}
& O\left(f_{k}\right)=Y\left(f_{k}\right)+\eta\left(f_{k}\right)=H\left(f_{k}\right) R\left(f_{k}\right)+\eta\left(f_{k}\right) \\
& D\left(f_{k}\right)=R\left(f_{k}\right)+\varepsilon\left(f_{k}\right)
\end{aligned}
$$

where, for example, $R\left(f_{k}\right)=\Delta t \sum_{t=0}^{N-1} r_{t} e^{-i 2 \pi k t / N}$. The convolution $r * h_{t}$ is now interpreted as cyclic due to the finite data length. Brillinger [16] points out that for this model the variates will be approximately uncorrelated at the Fourier frequencies, and "because of this weak correlation we can now consider applying the various classical procedures for approaching the problem of errors in variables. The solution ... will involve separate errors in variables solution for each of a number of frequencies" lying in $[0,1 /(2 \Delta t)]$. Note though, that in order to do this using, say, the regression maximum likelihood estimator [11], [15], we would need to know the input-output noise ratio in each of the chosen frequency ranges. Likewise, if we used the observable $D\left(f_{k}\right)$ as an instrument in an instrumental variables approach the input-output noise ratio is still required [17, p. 61].

Other possible approaches to the problem involve using higher order statistics [18]-[22]. The reflection coefficient series is known to have typically a non-Gaussian, but symmetric distribution [5]. Hence, for example in the frequency domain approach given in [22], the integrated trispectrum would be required. Also, as emphasized in [22], "higher-order statistics based methods typically yield high-variance estimates requiring 'large' record sizes to reduce the variance." One of the features of the geophysical data discussed above is that the time window $W$, over which the stationarity assumption is not obviously violated, typically contains between 200 and 400 sample values, a too small size to contemplate higherorder statistics. Our approach is thus appealing in using only second-order statistics and is also insensitive to the type or degree of non-Gaussianity of the reflection coefficient series.

\section{Multiple Coherence and Signal-TO-Noise Ratios}

Consider Figs. 1 and 2 again. So far we have considered the single location $P$ where first a seismic time series $\left\{O_{t}\right\}$ was recorded [Fig. 2(a)], and later a well was drilled and $\left\{D_{t}\right\}$ obtained [Fig. 2(b)]. However, during the survey represented by Fig. 2(a) the ship moves along a line firing off an explosion typically every $25 \mathrm{~m}$. Hence a series like $\left\{O_{t}\right\}$ will have been recorded every $25 \mathrm{~m}$ also. Let us consider $L=3$ such series recorded in the vicinity of the well location $P$, namely, one on either side of the well, and one at the well location $P$. For convenience we shall number the series at the well location as 1 and those on either side as 2 and 3. Thus, we are left with the following model for series $j$ :

$$
\begin{gathered}
O_{t, j}=y_{t, j}+\eta_{t, j}=\Delta t \sum_{k=-\infty}^{\infty} h_{k, j} r_{t-k}+\eta_{t, j} \\
j=1,2,3 .
\end{gathered}
$$

Note in particular that $\left\{O_{t, 1}\right\} \equiv\left\{O_{t}\right\}$. This model assumes that the series of reflection coefficients is the same at all $L$ locations since these are so close together, clustered around the well. The model also assumes that the signals on each series $\left\{y_{t, j}\right\}$ differ by a linear filtering only. Hence, the signal correlates from channel to channel. We assume the noise does not correlate from channel to channel and is uncorrelated with the signal. Let $S_{O}(f)$ be the full (Hermitian) cross-spectral matrix

$$
S_{O}=\left(\begin{array}{cc}
S_{O_{1}}(f) & S_{O_{1} O_{1}}(f) \\
S_{O_{1} O_{1}}(f) & S_{O_{1} O_{1}}(f)
\end{array}\right)
$$

where $S_{O_{1} O_{11}}(f)$ is the cross-spectral matrix of all the series $\left\{O_{t, j}\right\}$, excluding the first, $j=1$. Also, $S_{O_{1} O_{1}}(f)$ is the vector of cross-spectra of the $L-1$ series (the first being excluded) with the first

$$
S_{O_{1} O_{1}}(f)=\left[S_{O_{2} O_{1}}(f), S_{O_{3} O_{1}}(f), \cdots, S_{O_{L} O_{1}}(f)\right]
$$


and $S_{O_{1} O_{1}}(f)=S_{O_{1} O_{1}}^{H}(f)$, with ' $H$ ' denoting complexconjugate transpose. Let us denote the top-left element of the inverse of $S_{O}(f)$ by $S^{11}(f)$. Then

$$
S^{11}(f)=\left[S_{O_{1}}(f)-S_{O_{1} O_{/ 1}}(f) S_{O_{/ 1} O_{/ 1}}^{-1}(f) S_{O_{/ 1} O_{1}}(f)\right]^{-1} .
$$

Suppose we wish to predict series 1 by filtering the other $L-1$ series and adding the resultants together. (This is analogous to ordinary regression, except here filters play the roles of regression coefficients.) Wiener's minimum mean square error criterion states that the filters should be chosen so as to minimize the mean square error of the prediction. It follows [23, pp. 204-205 and 485-486] that the frequency response of the multichannel filter takes the (regression-like) form

$$
G_{O_{1} \mid O_{/ 1}}(f)=S_{O_{/ 1} O_{/ 1}}^{-1}(f) S_{O_{/ 1} O_{1}}(f) .
$$

The residual power spectrum from the least squares fit is then got by subtracting the prediction of $S_{O_{1}}(f)$, namely, $S_{O_{1} / O_{11}}(f) G_{O_{1} \mid O_{11}}(f)$, from $S_{O_{1}}(f)$

$$
S_{O_{1} \mid O_{1}}(f)=S_{O_{1}}(f)-S_{O_{1} O_{/ 1}}(f) S_{O_{/ 1} O_{/ 1}}^{-1}(f) S_{O_{/ 1} O_{1}}(f) .
$$

From (15) and (16), $S_{O_{1} \mid O_{11}}(f)=\left[S^{11}(f)\right]^{-1}$. Thus the fraction of power on series $\left\{O_{t, 1}\right\} \equiv\left\{O_{t}\right\}$ predicted from the other $L-1$ series at frequency $f$, known as the magnitude squared multiple coherence, may be written

$$
\gamma_{1}^{2}(f)=\frac{S_{O_{1}}(f)-S_{O_{1} \mid O_{/ 1}}(f)}{S_{O_{1}}(f)}=1-\left[S_{O_{1}}(f) S^{11}(f)\right]^{-1} .
$$

In the same way it follows that for series $\left\{O_{t, j}\right\}$

$$
\gamma_{j}^{2}(f)=\frac{S_{O_{j}}(f)-S_{O_{j} \mid O_{j}}(f)}{S_{O_{j}}(f)}=1-\left[S_{O_{j}}(f) S^{j j}(f)\right]^{-1}
$$

where $S^{j j}(f)$ is the $j$ th diagonal element of the inverse of the full cross-spectral matrix $S_{O}(f)$.

From (14), each of the $\left\{O_{t, j}\right\}$ have a spectrum $S_{O_{j}}(f)=$ $S_{y_{j}}(f)+S_{\eta_{j}}(f)$, with SNR given by $\rho_{j}(f)=S_{y_{j}}(f) / S_{\eta_{j}}(f)$, so that $S_{O_{j}}(f)=\left[1+\rho_{j}(f)\right] S_{\eta_{j}}(f)$. Since $\left\{O_{t, 1}\right\} \equiv\left\{O_{t}\right\}$ it follows that $\rho_{1}(f) \equiv \rho_{\text {out }}(f)$. Hence an estimate of $\rho_{1}(f)$ is identical to an estimate of $\rho_{\text {out }}(f)$.

Now the $j$ th diagonal element of the inverse of the full cross-spectral matrix $S_{O}(f)$ will be given by its $j$ th diagonal cofactor $\Delta_{/ j}(f)$, say, divided by its full determinant $\Delta(f)$. Hence, from (17)

$$
\gamma_{j}^{2}(f)=1-\frac{\Delta(f)}{\left[1+\rho_{j}(f)\right] S_{\eta_{j}}(f) \Delta_{/ j}(f)} .
$$

White [24] showed that

$$
\Delta(f)=S_{\eta_{1}}(f) \cdots S_{\eta_{L}}(f)\left[1+\rho_{1}(f)+\cdots+\rho_{L}(f)\right]
$$

and

$$
\Delta_{/ j}(f)=S_{\eta_{1}}(f) \cdots S_{\eta j-1}(f) S_{\eta j+1}(f) \cdots S_{\eta_{L}}(f) V(f)
$$

where

$$
V(f)=\left[1+\rho_{1}(f)+\cdots+\rho_{j-1}(f)+\rho_{j+1}(f)+\cdots+\rho_{L}(f)\right]
$$

which gives for $j=1, \cdots, L$

$$
\gamma_{j}^{2}(f)=\left(\frac{\rho_{j}(f)}{1+\rho_{j}(f)}\right)\left(\frac{\sum_{i=1}^{L} \rho_{i}(f)-\rho_{j}(f)}{1+\sum_{i=1}^{L} \rho_{i}(f)-\rho_{j}(f)}\right) .
$$

Hence, given good estimates of $\gamma_{j}^{2}(f), j=1, \cdots, L$ an iterative scheme should give estimates of $\rho_{j}(f), j=1, \cdots, L$. Only $\rho_{1}(f) \equiv \rho_{\text {out }}(f)$ is required. Estimation is discussed in the next section.

\section{The Estimation Scheme}

To summarize, our scheme consists of the following steps, carried out at each Fourier frequency, $f_{j}=j /(N \Delta t)$.

1) Estimate all the entries of the $L \times L$ cross-spectral matrix. From this, obtain estimates of $\gamma_{j}^{2}(f), j=1, \cdots, L$ using (17). Solve (18) for estimates of the SNR's $\rho_{j}(f), j=$ $1, \cdots, L$. Keep in particular the estimate of $\rho_{1}(f) \equiv$ $\rho_{\text {out }}(f)$.

2) Using estimates of the spectra $S_{D O}(f), S_{D}(f), S_{O}(f)$ of the observed series, estimate $\gamma_{D O}^{2}(f)$ from (9), and hence estimate $H(f)$ from (12).

The impulse response sequence $\left\{h_{t}\right\}$ is then found by inverse Fourier transformation. We now give a few details of how we implemented each of these estimation steps. The actual parameter values used which influence the estimation will be discussed in Section VII.

\section{A. Estimation Step One}

The cross-spectral matrix was estimated using the lag window cross-spectral estimator applied to the time series after tapering. The tapering helps reduce side-lobe leakage caused by the finite data lengths available. Let $\left\{d_{t}\right\}$ be a data taper standardized so that $\sum_{t=1}^{N} d_{t}^{2}=1$. For series of length $N$ the $j, k$ th entry of the cross-spectral matrix at frequency $f$ is

$$
\begin{aligned}
\hat{S}_{O_{j} O_{k}}^{(l w)}(f) & \equiv \int_{-f_{(N)}}^{f_{(N)}} W_{m}(f-\phi) \hat{S}_{O_{j} O_{k}}^{(d)}(\phi) d \phi \\
& =\Delta t \sum_{\tau=-(N-1)}^{N-1} w_{\tau, m} \hat{s}_{\tau, O_{j} O_{k}}^{(d)} e^{-i 2 \pi f \tau \Delta t}
\end{aligned}
$$

where the lag window $\left\{w_{\tau, m}\right\}$ and the smoothing window $W_{m}(\cdot)$ are Fourier transform pairs, with $w_{\tau, m}=0$ for $|\tau| \geq$ $N$, i.e., where $W_{m}(\cdot)$ is the smoothing window corresponding to the chosen lag window, i.e.,

$$
W_{m}(f)=\Delta t \sum_{\tau=-(N-1)}^{N-1} w_{\tau, m} e^{-i 2 \pi f \tau}
$$


and $m$ is a smoothing parameter. Here $\hat{S}_{O_{j} O_{k}}^{(d)}(f)$ is the direct cross-spectrum estimator, and $\hat{s}_{\tau, O_{j} O_{k}}^{(d)}$ is the estimator of the cross-covariance sequence (CCVS), both incorporating tapering. For $\tau=0, \cdots,(N-1)$ the CCVS estimator $\hat{s}_{\tau, O_{j} O_{k}}^{(d)}$ can be conveniently computed using

$$
\hat{s}_{\tau, O_{j} O_{k}}^{(d)}=\sum_{t=1}^{N-\tau} d_{t} O_{t, j} d_{t+\tau} O_{t+\tau, k}
$$

and

$$
\hat{s}_{\tau, O_{k} O_{j}}^{(d)}=\sum_{t=1}^{N-\tau} d_{t} O_{t, k} d_{t+\tau} O_{t+\tau, j}
$$

and when $\tau$ is negative, using the fact that $\hat{s}_{\tau, O_{j} O_{k}}^{(d)}=\hat{s}_{|\tau|, O_{k} O_{j}}^{(d)}$ and $\hat{s}_{\tau, O_{k} O_{j}}^{(d)}=\hat{s}_{|\tau|, O_{j} O_{k}}^{(d)}$. (Recall, the means of the series are known to be zero so no mean adjustments are required.) The Papoulis "minimum bias" lag window was used here (see e.g., [25, pp. 266-267]). Smoothing properties are controlled by the smoothing parameter $m$-spectral smoothing increases as $m$ decreases and as this happens the estimated CCVS becomes increasingly truncated. The number of complex degrees of freedom $n$ associated with such an estimator is given by (e.g., [25, Sect. 6.10])

$$
n=\nu / 2=\frac{N B_{w} \Delta t}{C_{d}}=\frac{N \Delta t}{C_{d} \int_{-f_{(N)}}^{f_{(N)}} W_{m}^{2}(\theta) d \theta}
$$

where $B_{W}=\left[\int_{-f_{(N)}}^{f_{(N)}} \quad W_{m}^{2}(\theta) d \theta\right]^{-1}$ is the definition of smoothing window bandwidth due to Jenkins [26] (see also [25, Sect. 6.8]) and $C_{d}$ is a variance inflation factor due to tapering which has the form $C_{d}=N \sum_{t=1}^{N} d_{t}^{4}$. For the Papoulis lag window

$$
n=1.7 N /\left(m C_{d}\right)
$$

The cross-spectral matrix must be invertible. If the number $n$ of complex degrees of freedom of the cross-spectral estimators are such that $n<L$, the matrix will be singular [24]. Also, given estimates of the $\gamma_{j}^{2}(f)$, for the $L$ series, estimates of the $\rho_{j}(f)$ can be determined only if $L>2$ [since when $L=2$ there is a single coherence and two unknown SNR's-see (11)]. Additional bias in coherence estimates can be caused by alignment problems, e.g., due to significantly different positions of a strong reflection event from one end of the block of $L$ contiguous series to the other. The choice $L=3$ is a good compromise in that in the real-world alignment changes should be negligible, while also putting no real limitation on the range of choices of smoothing $(n \geq 3$.)

Using (17) estimates $\hat{\gamma}_{j}^{2}(f), j=1, \cdots, L$ were obtained from the entries in the cross-spectral matrix. Maximumlikelihood estimates of these magnitude-squared multiple coherencies were debiased in the standard way (e.g., [24])

$$
\tilde{\gamma}_{j}^{2}(f) \approx \frac{\hat{\gamma}_{j}^{2}(f)-[(L-1) / n]}{1-[(L-1) / n]} .
$$

Estimated SNR's $\hat{\rho}_{j}(f), j=1, \cdots, L$ were found by iteratively solving the implicit equations (18)

$$
\hat{K}_{j}(f) \tilde{\gamma}_{j}^{2}(f)=\hat{\rho}_{j}(f) /\left[1+\hat{\rho}_{j}(f)\right]
$$

where

$$
\hat{K}_{j}(f)=\left[1+\sum_{i=1}^{L} \hat{\rho}_{i}(f)-\hat{\rho}_{j}(f)\right] /\left[\sum_{i=1}^{L} \hat{\rho}_{i}(f)-\hat{\rho}_{j}(f)\right] .
$$

To end step 1 we set $\hat{\rho}_{\text {out }}(f)=\hat{\rho}_{1}(f)$.

\section{B. Estimation Step Two}

The spectrum $S_{O}(f)$ is identical to the spectrum $S_{O_{1}}(f)$, since $\left\{O_{t}\right\} \equiv\left\{O_{t, 1}\right\}$. Thus the smoothed spectrum estimate $\hat{S}_{O}(f)$ is already available from step 1 . The cross-spectrum $S_{D O}(f)$ and the spectrum $S_{D}(f)$ were additionally estimated by the lag-window method detailed in step 1 . However, crossspectral estimates are subject to misalignment bias: this occurs if there is a large phase difference between the series, as can happen when energy peaks are not aligned. In step 1 this was not a problem because all $L$ series are essentially replications of the same thing, due to their close proximity. However, energy peaks between $\left\{D_{t}\right\}$ and $\left\{O_{t}\right\}$ can occur at different times due to the effect of the filter $\left\{h_{t}\right\}$. The standard solution is, having computed the cross-covariance sequence $\hat{s}_{\tau, D O}^{(d)}$, to shift the series until the peak of the sequence is at zero lag; the shift is noted and reapplied to the impulse response estimate.

Having estimated $\hat{\gamma}_{D O}^{2}(f)$ from (9), it is also debiased by converting it to $\tilde{\gamma}_{D O}^{2}(f)$ using (24) with $L=2$, the ordinary coherence correction [24].

Finally, we obtain our estimate $\hat{H}(f)$ of the frequency response function

$$
\hat{H}(f)= \begin{cases}\frac{1}{\tilde{\gamma}_{D O}^{2}(f)}\left[\frac{\hat{\rho}_{\text {out }}(f)}{1+\hat{\rho}_{\text {out }}(f)}\right] \hat{H}_{O \mid D}(f), & \text { if } \hat{\rho}_{\text {out }}(f)>0 \\ 0, & \text { otherwise. }\end{cases}
$$

\section{Relative Variances}

The forms of (5)-(7) mean that we can also formulate the filter that predicts $\left\{D_{t}\right\}$ from $\left\{O_{t}\right\}$ in the least squares sense as

$$
\begin{aligned}
H_{D \mid O}(f) & \equiv \frac{S_{O D}(f)}{S_{O}(f)}=\frac{S_{y r}(f)}{S_{y}(f)+S_{\eta}(f)} \\
& =\frac{S_{y r}(f)}{|H(f)|^{2} S_{r}(f)+S_{\eta}(f)} .
\end{aligned}
$$

Hence, if $S_{\eta}(f) \equiv 0$, then

$$
H_{D \mid O}(f)=\frac{H^{*}(f) S_{r}(f)}{|H(f)|^{2} S_{r}(f)+S_{\eta}(f)}=[H(f)]^{-1} .
$$

The two cases: 1) $S_{\varepsilon}(f) \equiv 0$, so that the input SNR is infinite, or $\lambda(f)=0$, and $H_{O \mid D}(f) \equiv H(f)$, and 2) $S_{\eta}(f) \equiv 0$, so that the output SNR is infinite, or $\lambda(f)=\infty$, and 


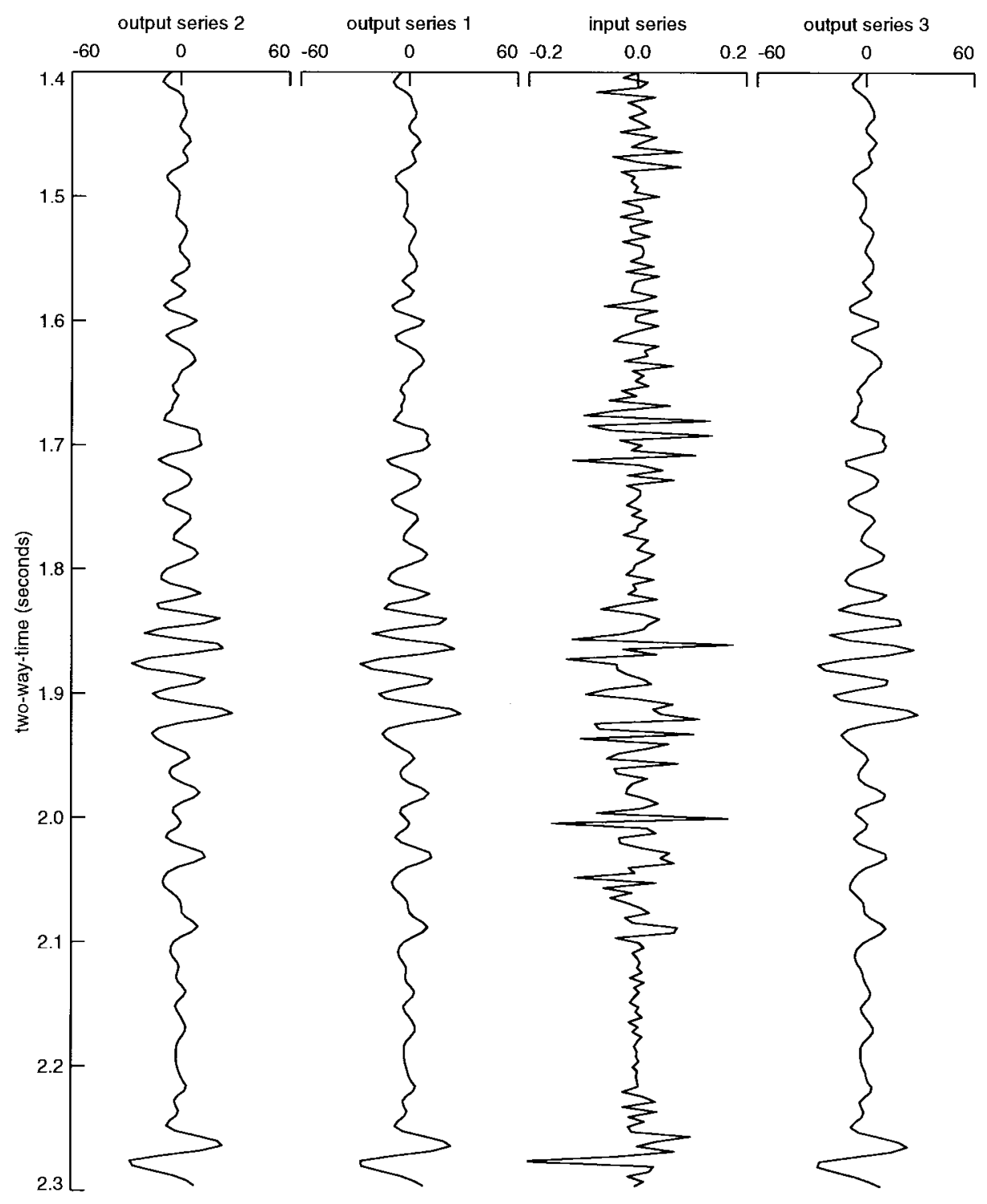

Fig. 3. Three seismic reflection time series $\left\{O_{t, 1}\right\},\left\{O_{t, 2}\right\}$, and $\left\{O_{t, 3}\right\}$, recorded during a survey in the southern North Sea, and labeled "output series" 1,2 , or 3 . Series 1 is at the well location. Also shown is the approximate reflection sequence $\left\{D_{t}\right\}$, labeled "input series," derived at the well. The sample interval is $\Delta t=4 \mathrm{~ms}$ so that 225 sample values are shown.

$H_{D \mid O}(f) \equiv[H(f)]^{-1}$, cover the two possible degenerate noise structures corresponding to (5)-(7). A third interesting case is when $\lambda(f)=S_{\varepsilon}(f) / S_{\eta}(f)=S_{D}(f) / S_{O}(f)$, i.e., the noise spectrum ratios are equal to the total input/output spectrum ratios. Substituting in (13) we obtain

$$
H(f)=\left[\frac{S_{O}(f)}{S_{D}(f)}\right]^{1 / 2} \frac{S_{D O}(f)}{\left|S_{D O}(f)\right|} .
$$

This is identical to taking the geometric mean of the two degenerate noise cases, since

$$
\begin{aligned}
H^{2}(f) & =\left[\frac{H_{O \mid D}(f)}{H_{D \mid O}(F)}\right]=\frac{S_{O}(f)}{S_{D}(f)} \frac{S_{D O}(f)}{S_{D O}^{*}(f)} \\
& =\frac{S_{O}(f)}{S_{D}(f)} \exp \left\{i 2 \arg \left[S_{D O}(f)\right]\right\} .
\end{aligned}
$$

The spectral errors propagate into the estimates of the frequency response function. The asymptotic relative variance of the amplitude response $\left|\hat{H}_{O \mid D}(f)\right|$ is given by [10]

$$
\frac{\operatorname{var}\left\{\left|\hat{H}_{O \mid D}(f)\right|\right\}}{\left|H_{O \mid D}(f)\right|^{2}}=\frac{\gamma_{D O}^{-2}(f)-1}{2 n}
$$

and the same expression is appropriate for $\left|\hat{H}_{D \mid O}(f)\right|$ and for the phase responses. The relative variance for the estimated amplitude response corresponding to (23) is given by [27]

$$
\frac{\operatorname{var}\{|\hat{H}(f)|\}}{|H(f)|^{2}}=\frac{1-\gamma_{D O}^{2}(f)}{2 n} .
$$

The expressions (24) and (25) set limits on the relative variance of the estimated amplitude response (22); the general expression for relative variance is very complicated. However, (24) and (25) suffice to show how the coherence, $\gamma_{D O}^{2}(f)$ and the number of complex degrees of freedom $n$ affect the relative variances. 

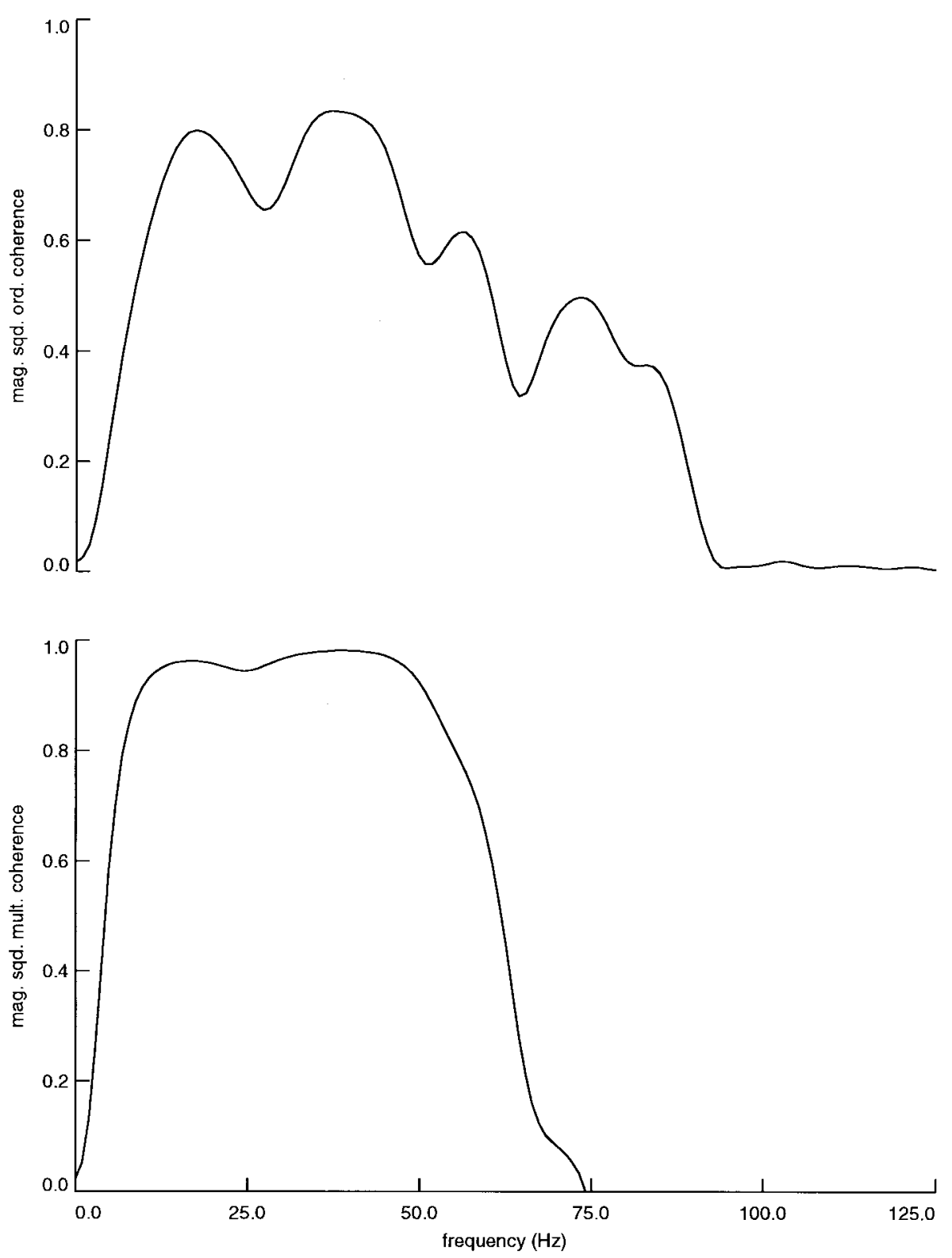

Fig. 4. The estimated ordinary coherence (top) between input and output $\tilde{\gamma}_{D O}^{2}(f)$, and the estimated magnitude squared multiple coherence (bottom) at the well location, $\tilde{\gamma}_{1}^{2}(f)$, at the Fourier frequencies.

\section{EXAMPLE RESULTS}

To test and demonstrate the method proposed above, we first apply the technique to real data recorded during a survey in the southern North Sea. This could be described as an uncontrolled case study. Second, we carry out a controlled experiment using simulated data and various SNR's on input and output.

\section{A. Real Data}

Three seismic reflection time series $\left\{O_{t, 1}\right\},\left\{O_{t, 2}\right\}$, and $\left\{O_{t, 3}\right\}$, and labeled "output series" 1,2 or 3 , are shown in Fig. 3. Series 1 is at the well location. Also shown is the approximate reflection sequence or "synthetic seismogram" $\left\{D_{t}\right\}$, labeled "input series," derived at the well. The gate of data shown starts at a two-way reflection time of $1.4 \mathrm{~s}$ and is of $900 \mathrm{~ms}$ duration; the sample interval is $\Delta t=4 \mathrm{~ms}$ so that 225 samples are available. Note that the input series is of a higher frequency content than the output series; $H(f)$ acts as a lowpass filter. This makes sense, since the "input series" represents the reflection sequence in situ, while the "output series" are recorded at the sea surface and contain the effect of the two-way passage of the seismic explosion down to the depth of interest and back to the surface. Since the layers of the Earth preferentially absorb high frequencies, the frequency response will essentially be lowpass in form.

The seismic time series form part of a "stacked section" and are the result of several processing steps, including the removal of water-bottom multiples using predictive deconvolution [2] and the enhancement of primary reflections using beamforming [28]. 


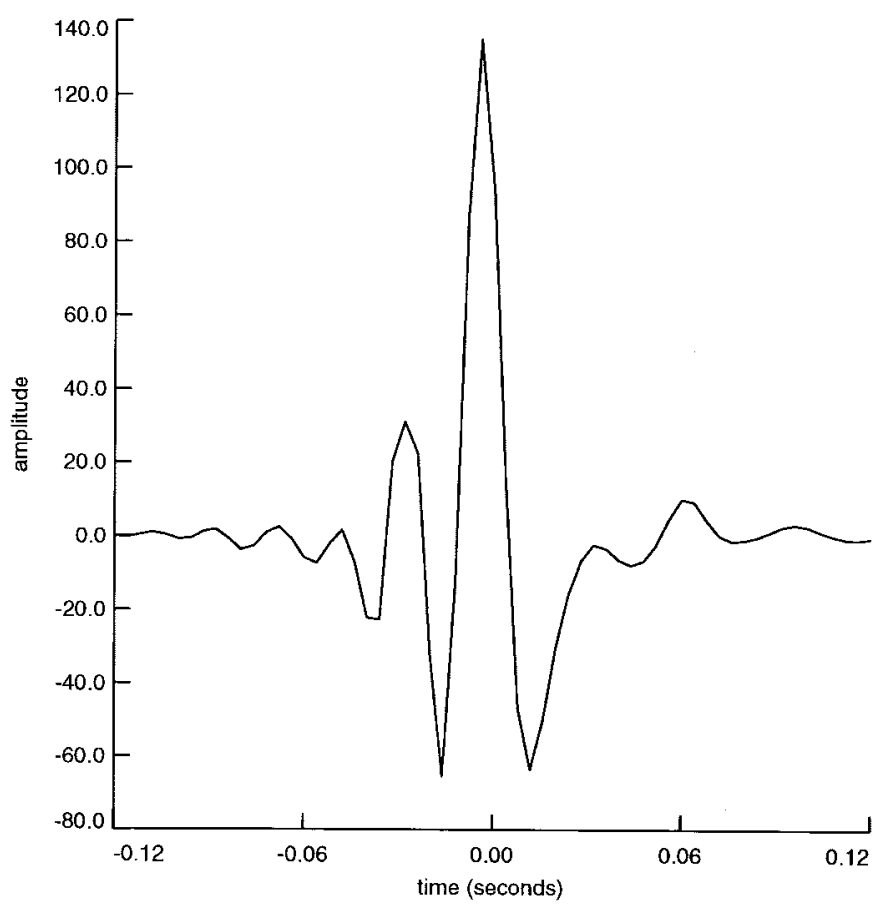

Fig. 5. The estimated impulse response (seismic wavelet) $\left\{\hat{h}_{t}\right\}$. Note the significant energy before time zero.

A $20 \%$ cosine taper was applied to all series; for such a taper $C_{d}=1.12$. The smoothing parameter $m$ was set to 32 so that from (20), there are $n=10.7$ effective complex degrees of freedom at each frequency. (This choice is guided by results from simulating the effects of different smoothing levels on estimating seismic wavelets and many years' experience with seismic wellties. It represents a good tradeoff between bias from spectral smoothing and the suppression of random error in the wavelet estimate.)

The estimated ordinary coherence between input and output $\tilde{\gamma}_{D O}^{2}(f)$ and the estimated magnitude squared multiple coherence at the well location $\tilde{\gamma}_{1}^{2}(f)$ are shown in Fig. 4 at the Fourier frequencies. We note in particular the high multiple coherence across the seismic bandwidth.

Fig. 5 gives the estimated impulse response or seismic wavelet $\left\{\hat{h}_{t}\right\}$. The convolution of $\left\{\hat{h}_{t}\right\}$ with the observed input series $\left\{D_{t}\right\}$ accounts for $75 \%$ of the power in the observed output series $\left\{O_{t, 1}\right\}$ at the well location. (Recall that in physical reality $\left\{\hat{h}_{t}\right\}$ is convolved with unobserved true input series $\left\{r_{t}\right\}$.) Note that there is much significant energy in the estimated seismic wavelet before time zero, so that the wavelet is clearly not minimum-delay; hence, as expected, methods for its estimation which assume minimum-delay, such as [13], would not work for this data. The presence of anticipation terms is typical of a seismic waveform present on seismic data, where they arise from shifts due to the recording equipment, and effects caused by the many other preliminary processing steps. One of the authors has investigated ARMA modeling to seismic wavelet estimation and concluded that because of the noncausality of wavelets in processed data and their inherently strongly bandlimited spectra, these methods are neither as

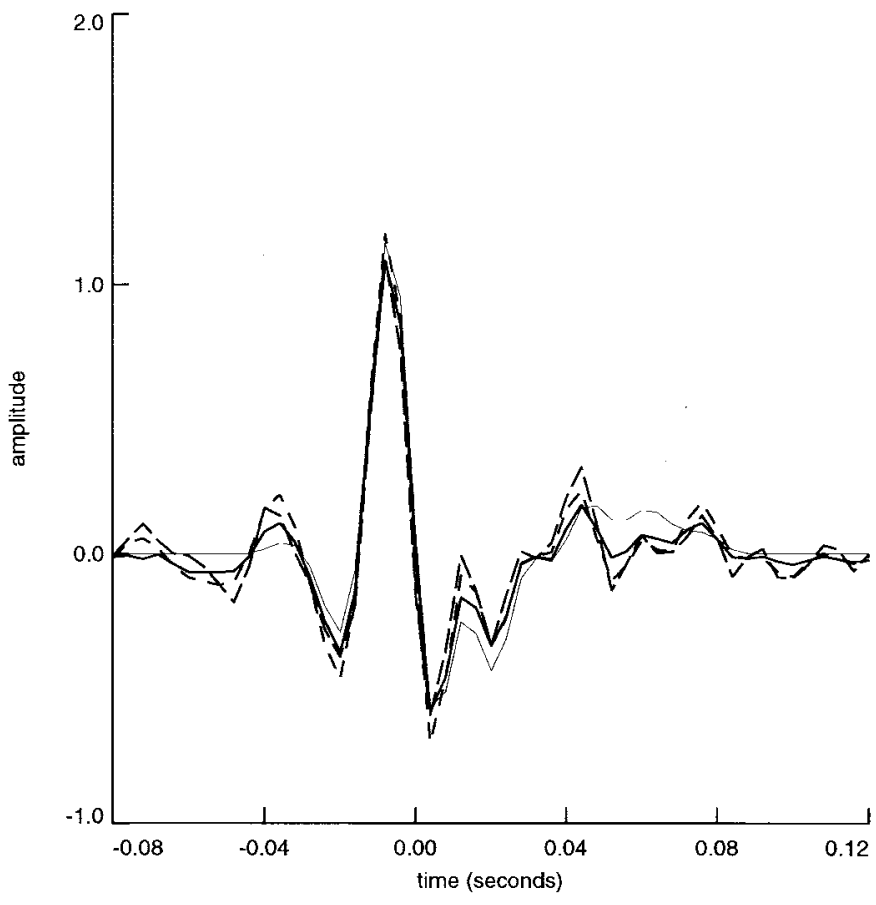

Fig. 6. True impulse response (thin line) and estimates when input and output signal-to-noise variance ratios are (a) both 10:1 (solid line), (b) 2:1 and 10:1, respectively, (line with short dashes), and (c) both 2:1 (line with long dashes).

convenient nor as effective as a nonparametric approach, such as used here.

\section{B. Simulated Data}

For the simulation process a reflectivity sequence $\left\{r_{t}\right\}$ (with $N=400$ points) and an impulse response sequence $\left\{h_{t}\right\}$ shown in Fig. 6 were used. Both this reflectivity series and impulse response sequence are themselves estimates from a physical experiment since we can never measure precisely the seismic waveform or reflectivity inside the earth. This is true remote sensing. However, these estimates have all the typical characteristics, and so are ideal for simulation purposes. Note that the impulse response consists of values before time zero (anticipation terms) and values after time zero. The sample interval is the typical value of $\Delta t=4 \mathrm{~ms}$, giving a Nyquist frequency of $f_{(N)}=1 /(2 \Delta t)=125 \mathrm{~Hz}$. To synthesize $\left\{D_{t}\right\}$, uncorrelated white Gaussian noise was added to $\left\{r_{t}\right\}$. The output series $\left\{O_{t}\right\} \equiv\left\{O_{t, 1}\right\}$ was synthesized by adding white Gaussian noise to $\left\{y_{t}\right\}$. The other output series $\left\{O_{t, 2}\right\},\left\{O_{t, 3}\right\}$, and $\left\{O_{t, 4}\right\}$ were created similarly, using independent noise realizations.

Three experiments were carried out. In the first the input signal-to-noise variance ratio $\int S_{r}(f) d f / \int S_{\varepsilon}(f) d f$ and the output signal-to-noise variance ratio $\int S_{y}(f) d f / \int S_{\eta}(f) d f$ were both set to 10:1. Practical experience has shown that, in some cases, the series $\left\{D_{t}\right\}$, an approximation to $\left\{r_{t}\right\}$, acquired from a rough bore hole can be quite heavily in error. Hence, for the second experiment a 10:1 ratio on the output signal-to-noise was maintained, but this was reduced to a 2:1 ratio for input signal-to-noise. For the third experiment a 
pessimistic 2:1 variance ratio was specified for both the input and output signal-to-noises, which represents a severe test of the method.

Estimation was carried out using about $n=12$ complex degrees of freedom at each frequency in the spectral estimation of both input and outputs. The true impulse response $\left\{h_{t}\right\}$ is compared to the estimates from the three experiments in Fig. 6. As expected from the discussion of relative variances in Section VI, there is a degradation in estimate with decreasing signal-to-noise variance ratios, but even in the extremely pessimistic scenario of experiment three (2:1 ratios), the dominant features of the true impulse response are still to be seen in the estimate.

\section{CONCLUSIONS}

It has been demonstrated that the estimation of the impulse response in the geophysical noisy input-output (errors in variables) problem discussed here can be tackled by the combination of 1) multiple coherence analysis applied to the replicated time series with 2) ordinary coherence analysis applied to the observed input and output series. The key to the solution lies in the estimation of SNR's at each of the Fourier frequencies. (Although not discussed in detail here, it is possible to use this information also to estimate the spectra of the input and output noise from the spectra of the corresponding observed series and, hence, gain information on the nonwhiteness of the noise spectra.) No strong assumptions on the nature of the impulse response sequence, such as that it is minimum delay, have been made, nor is anything assumed about the level or types of non-Gaussianity of the series involved. Hence the method is generally applicable in this geophysical context. Accurate tying of seismic data $\left\{O_{t}\right\}$ to synthetic seismograms $\left\{D_{t}\right\}$ recorded at wells are increasingly important in modern seismic interpretation and virtually indispensable in reservoir geophysics which relies very heavily on the integration of "downhole" and seismic data. We believe that the quantitative methods discussed here could be indispensable in establishing the reliability of wellties.

\section{APPENDIX}

\section{EQuivalence to Classical EIV FoRMula}

We start with the nonnull case in (12). Substituting for $\gamma_{D O}^{2}(f)$ we get

$$
\begin{aligned}
H(f) & =\frac{S_{D}(f) S_{O}(f)}{\left|S_{D O}(f)\right|^{2}}\left[\frac{\rho_{\text {out }}(f)}{1+\rho_{\text {out }}(f)}\right] \frac{S_{D O}(f)}{S_{D}(f)} \\
& =\frac{S_{O}(f) S_{D O}(f)}{\left|S_{D O}(f)\right|^{2}}\left[\frac{\rho_{\text {out }}(f)}{1+\rho_{\text {out }}(f)}\right] .
\end{aligned}
$$

But

$$
\rho_{\text {out }}(f)=\frac{S_{y}(f)}{S_{\eta}(f)}=\frac{S_{O}(f)}{S_{\eta}(f)}-1
$$

and

$$
\rho_{\mathrm{in}}(f)=\frac{S_{r}(f)}{S_{\varepsilon}(f)}=\frac{S_{D}(f)}{S_{\varepsilon}(f)}-1
$$

Hence

$$
\begin{aligned}
\frac{\rho_{\text {out }}(f)}{1+\rho_{\text {out }}(f)}= & \frac{S_{\eta}(f)}{2 S_{O}(f)}\left\{\left[\rho_{\text {out }}(f)-\rho_{\text {in }}(f)\right]\right. \\
& \left.+\left[\rho_{\text {out }}(f)+\rho_{\text {in }}(f)\right]\right\} \\
= & \frac{S_{\eta}(f)}{2 S_{O}(f)}\left\{\left[\rho_{\text {out }}(f)-\rho_{\text {in }}(f)\right]\right. \\
& \left.+\sqrt{\left[\rho_{\text {out }}(f)-\rho_{\text {in }}(f)\right]^{2}+4 \rho_{\text {in }}(f) \rho_{\text {out }}(f)}\right\} \\
= & \frac{S_{\eta}(f)}{2 S_{O}(f)}\left\{\left[\frac{S_{O}(f)}{S_{\eta}(f)}-\frac{S_{D}(f)}{S_{\varepsilon}(f)}\right]\right. \\
& +\sqrt[+]{\left.\left[\frac{S_{O}(f)}{S_{\eta}(f)}-\frac{S_{D}(f)}{S_{\varepsilon}(f)}\right]^{2}+4 \frac{S_{r}(f) S_{y}(f)}{S_{\varepsilon}(f) S_{\eta}(f)}\right\}} .
\end{aligned}
$$

Put $\lambda(f)=S_{\varepsilon}(f) / S_{\eta}(f)$, and set $U(f)$ equal to

$$
\sqrt[t]{\left[\lambda(f) S_{O}(f)-S_{D}(f)\right]^{2}+r\left[S_{r}(f) S_{y}(f) S_{\varepsilon}(f) / S_{\eta}(f)\right]} .
$$

Then

$$
\frac{\rho_{\text {out }}(f)}{1+\rho_{\text {out }}(f)}=\frac{\left\{\left[\lambda(f) S_{O}(f)-S_{D}(f)\right]+U(f)\right.}{2 \lambda(f) S_{O}(f)} .
$$

But $S_{r}(f) S_{y}(f)=\left|S_{r y}(f)\right|^{2}=\left|S_{D O}(f)\right|^{2}$, so that

$$
U(f)=\sqrt{\left[\lambda(f) S_{O}(f)-S_{D}(f)\right]^{2}+4 \lambda(f)\left|S_{D O}(f)\right|^{2}}
$$

and

$$
\begin{aligned}
H(f) & =\frac{S_{D O}(f)\left\{\left[\lambda(f) S_{O}(f)-S_{D}(f)\right]+U(f)\right\}}{2 \lambda(f)\left|S_{D O}(f)\right|^{2}} \\
& =\frac{\left\{\left[\lambda(f) S_{O}(f)-S_{D}(f)\right]+U(f)\right\}}{2 \lambda(f) S_{D O}^{*}(f)} .
\end{aligned}
$$

This form is similar to that given by Pisarenko [29], who does not however set it in the context of regression and spectral coherence.

\section{ACKNOWLEDGMENT}

The authors are extremely grateful to Fina Exploration, Ltd. for permission to use their data for the illustrative example.

\section{REFERENCES}

[1] E. A. Robinson, Physical Applications of Stationary Time-Series. London, U.K.: Charles Griffin, 1980.

[2] E. A. Robinson and S. Treitel, Geophysical Signal Analysis. Englewood Cliffs, NJ: Prentice-Hall, 1980

[3] A. T. Walden and J. W. J. Hosken, "Choosing the block averaging interval when calculating primary reflection coefficients from well logs," Geophys. Prosp., vol. 36, pp. 799-824, 1988.

[4] "An investigation of the spectral properties of primary reflection coefficients," Geophys. Prosp., vol. 33, pp. 400-435, 1985.

[5] , "The nature of the non-Gaussianity of primary reflection coefficients and its significance for deconvolution," Geophys. Prosp., vol. 34, pp. 1038-1066, 1986.

[6] R. H. Shumway and Z. A. Der, "Deconvolution of multiple time series," Technometrics, vol. 27, pp. 385-393, 1985.

[7] G. M. Webster, Ed., Deconvolution. Tulsa, OK: Soc. Exploration Geophys., 1978.

[8] R. E. White, "Partial coherence matching of synthetic seismograms with seismic traces," Geophys. Prosp., vol. 28, pp. 333-358, 1980.

[9] A. T. Walden and R. E. White, "On errors of fit and accuracy in matching synthetic seismograms and seismic traces," Geophys. Prosp., vol. 32, pp. 871-891, 1984. 
[10] R. E. White, "Signal and noise estimation from seismic reflection data using spectral coherence methods," Proc. IEEE, vol. 72, pp. 1340-1356, 1984.

[11] G. Casella and R. L. Berger, Statistical Inference. Belmont, CA: Duxbury, 1990

[12] M. J. Hinich, "Estimating the gain of a linear filter from noisy data," in Handbook of Statistics, Volume 3, D. R. Brillinger and P. R. Krishnaiah, Eds. Amsterdam, The Netherlands: Elsevier, 1983, pp. 157-167.

[13] M. J. Hinich and W. E. Weber, "Estimating linear filters with errors in variables using the Hilbert transform," Signal Process., vol. 37, pp. 215-228, 1994.

[14] R. E. White, "Estimation problems in seismic deconvolution," in Proc. 1986 Res. Workshop Deconvolution Inversion, M. Bernabini et al., Eds. Oxford, U.K.: Blackwell, 1987, pp. 5-37.

[15] W. A. Fuller, Measurement Error Models. New York: Wiley, 1987.

[16] D. R. Brillinger, Time Series, Data Analysis and Theory, Expanded Edition. New York: McGraw-Hill, 1981.

[17] R. J. Bowden and D. A. Turkington, Instrumental Variables. Cambridge, U.K.: Cambridge Univ. Press, 1984

[18] J. K. Tugnait, "Stochastic system identification with noisy input using cumulant statistics," IEEE Trans. Automat. Contr., vol. 37, pp. 476-485, 1992.

[19] Y. Inouye and H. Tsuchiya, "Identification of linear systems using input-output cumulants," Int. J. Contr., vol. 53, 1431-1448, 1991.

[20] A. Delopoulos and G. B. Giannakis, "Consistent identification of stochastic linear systems with noisy input-output data," Automatica, vol. 30, pp. 1271-1294, 1994.

[21] Y. Inouye and Y. Suga, "Identification of linear systems with noisy input using input-output cumulants," Int. J. Contr., vol. 59, 1231-1253, 1994.

[22] J. K. Tugnait and Y. Ye, "Stochastic system identification with noisy input-output measurements using polyspectra," IEEE Trans. Automat. Contr., vol. 40, pp. 670-683, 1995.

[23] G. M. Jenkins and D. G. Watts, Spectral Analysis and Its Applications. San Francisco, CA: Holden-Day, 1968.

[24] R. E. White, "The estimation of signal spectra and related quantities by means of the multiple coherence function," Geophys. Prosp., vol. 21, pp. 660-703, 1973.

[25] D. B. Percival and A. T. Walden, Spectral Analysis for Physical Applications. Cambridge, U.K.: Cambridge Univ. Press, 1993.

[26] G. M. Jenkins, "General considerations in the analysis of spectra," Technometrics, vol. 3, pp. 133-166, 1961.

[27] S. A. Raikes and R. E. White, "Measurements of earth attenuation from downhole and surface seismic recordings," Geophys. Prosp., vol. 32, pp. 892-919, 1984

[28] T. Hu and R. E. White, "Implementation of MVU beamformers," in Exp. Abstr. 55th EAEG Meeting, Stavanger, Norway, 1993, paper P013.

[29] V. F. Pisarenko, "Statistical estimates of amplitude and phase corrections," Geophys. J. Roy. Astron. Soc., vol. 20, pp. 89-98.

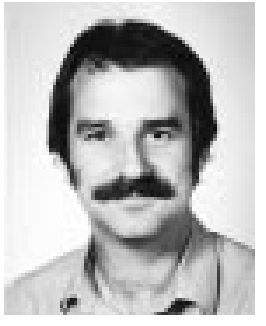

Andrew T. Walden (A'86) received the B.Sc. degree in mathematics from the University of Wales, Cardiff, U.K., in 1977 and the M.Sc. and Ph.D. degrees in statistics from the University of Southampton, Southampton, U.K., in 1979 and 1982.

He was a Research Scientist at British Petroleum from 1981 to 1990, specializing in statistical signal processing for oil and gas exploration. He spent 1985 and 1986 as a Visiting Assistant Professor at the University of Washington, Seattle, teaching and researching in both the Statistics and Geophysics Departments. He joined the Department of Mathematics, Imperial College, London, U.K., in 1990, where he is currently Professor of Statistics. His research interests are in time series, spectrum analysis, and wavelets, with applications to geophysics and oceanography. He coauthor, with D. Percival, of the text Spectral Analysis for Physical Applications (Cambridge, U.K. Cambridge Univ. Press, 1993).

Dr. Walden received the Van Weelden Award of the European Association of Geoscientists and Engineers in 1984

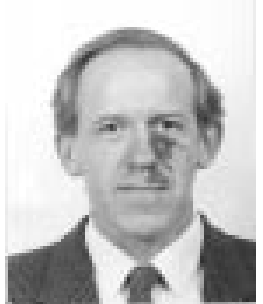

Roy E. White received the B.A. degree in 1959, the Cert. Ed. degree in 1960, and the M.A. degree in 1963, all from the University of Cambridge, Cambridge, U.K., and the Ph.D. degree in 1969 from the University of Adelaide, Adelaide, Australia.

In 1960, he was appointed Demonstrator in Physics at the University of Adelaide, and from 1962 to 1968 was Lecturer in Physics at the South Australian Institute of Technology. After two and a half years as Postdoctoral Fellow and Lecturer in the Department of Geophysics at the University of Western Ontario, London, Ont., Canada, he joined British Petroleum Exploration in 1971 as a Research Geophysicist, becoming a Research Associate in 1979. He joined Birkbeck College, University of London, London, U.K., in 1989, where he maintains strong contacts with the oil industry through research in reservoir geophysics and petrophysics. He is currently Professor of Geology. He has a particular interest in the application of statistical methods to estimation problems and measuring accuracy in exploration seismology.

Dr. White is a recipient of the Conrad Schlumberger Award of the European Association of Geoscientists and Engineers and is an Honorary Member of the Society of Exploration Geophysicists. 